\title{
Subblock Processing for Frequency-Domain Turbo Equalization under Fast Fading Environments
}

\author{
Keiichi KAMBARA $^{\dagger \text { a) }}$, Hiroshi NISHIMOTO ${ }^{\dagger}$, Toshihiko NISHIMURA ${ }^{\dagger}$, Takeo OHGANE ${ }^{\dagger}$, \\ and Yasutaka OGAWA ${ }^{\dagger}$, Members
}

\begin{abstract}
SUMMARY Frequency-domain equalization (FDE) has been studied for suppressing inter-symbol interference (ISI) due to frequency selective fading in single carrier systems. When a high-mobility terminal is assumed in the system, channel transition within an FDE block cannot be ignored. The ISI reduction performance of FDE degrades since the cyclicity of the channel matrix is lost. To solve this problem, a method of dividing the received data block into multiple subblocks has been proposed, where pseudo cyclic prefix $(\mathrm{CP})$ processing is introduced to realize periodicity in each subblock. In this method, the performance is degraded by the inherentlyinaccurate pseudo CP. In this paper, we study the application of frequencydomain turbo equalization (FDTE) to subblock processing for improving the accuracy of pseudo CP. The simulation results show that FDTE with subblock processing yields remarkable performance improvements.

key words: fast fading, single carrier transmission, SC/MMSE-FDTE, unique word, subblock processing
\end{abstract}

\section{Introduction}

Currently, higher data-rate services greater than $100 \mathrm{Mbps}$ are discussed in the standardization process for beyond third generation systems. Such wideband systems suffer from frequency selective fading so that multi-carrier transmission, for example, orthogonal frequency division multiplexing (OFDM), is very effective [1]. However, OFDM systems have the underlying problem of high peak-to-averagepower-ratio (PAPR) [2]. Thus, the use of single carrier transmission for the uplink is mandatory when we consider the power consumption at the hand set.

While single carrier transmission has an advantage of low PAPR, a countermeasure to inter-symbol interference (ISI) due to frequency selective fading is required. Frequency-domain equalization (FDE) has been known as the simplest ISI reduction method in severe fading environments [3]. Unfortunately, FDE properly works under block fading channels only. Thus, in fast fading environments, channel transition within an FDE block degrades the equalization performance. Specifically, the cyclic matrix property of the channel matrix in the FDE is not satisfied so that the unequalized component causes the residual ISI in the FDE process.

A method of adaptively controlling the transmission block size has been proposed [4]. By changing the block

Manuscript received August 25, 2008.

Manuscript revised December 24, 2008.

$\dagger$ The authors are with the Graduate School of Information Science and Technology, Hokkaido University, Sapporo-shi, 0600814 Japan.

a)E-mail: kan@m-icl.ist.hokudai.ac.jp

DOI: 10.1587/transcom.E92.B.1466 size at the transmitter according to the instantaneous fading speed, it can mitigate the channel transition within the block. However, when the fading speed is very high, the reduced transmission block size with a constant cyclic prefix (CP) size results in a decrease in transmission efficiency. In addition, the transmitter needs to know information about the fading speed as a closed-loop system is required. Thus, we have proposed subblock FDE processing at the receiver side where the received block is divided into multiple subblocks [5], [6]. It has been clarified that the proposed subblock processing provides remarkable improvement in FDE performance. However, it has also been found that the performance is degraded by the inherently-inaccurate pseudo $\mathrm{CP}$, which is introduced to satisfy periodicity in a subblock FDE window approximately.

The frequency-domain turbo equalization (FDTE) [7][10] is known as a powerful technique to suppress the ISI effect. The FDTE reduces the ISI by the soft cancellation basically. Thus, the FDTE has a potential to work in a nonblock fading channel. However, high capability in the fast fading environment can not be expected since the cancellation error occurs in the top and the tail of the block due to channel change within the block. Thus, applying the subblock processing to the FDTE would be a good solution to improve the performance in the fast fading environment. In addition, the accuracy of the soft replicas for ISI cancellation are expected to be improved with iterations based on the turbo principle. This property is very suitable for pseudo $\mathrm{CP}$ generation in the subblock processing.

In the paper, the performance of the FDTE with the subblock processing is evaluated in non-block fading environments. The rest of the paper is organized as follows. In Sect. 2, fundamental formulation of FDE and FDTE are reviewed. Then, the proposed method is described in Sect. 3. Section 4 explains the channel estimation scheme using a known pilot sequence. After numerical analysis in Sect. 5, Sect. 6 concludes the paper.

\section{Frequency-Domain Turbo Equalization Under Fast Fading Environments}

\subsection{Conventional FDE Based on Cyclicity of Channel Ma- trix}

Let us consider a single carrier system with a block transmission of $N$ symbols and FDE at the receiver side. We 
define an $N$-dimensional transmit signal vector as $\boldsymbol{s}=$ $\left[s_{0}, \cdots, s_{N-1}\right]^{T}$ and assume a multipath channel with $L$ symbol-spaced paths $h_{0}, h_{1}, \ldots, h_{L-1}$.

Adding a proper $\mathrm{CP}$ longer than $(L-1)$ symbols at the transmitter side and discarding the $\mathrm{CP}$ part at the receiver side, we obtain the $N$-dimensional received signal vector over a block fading channel as

$$
\boldsymbol{r}=\boldsymbol{H} \boldsymbol{s}+\boldsymbol{n},
$$

where $\boldsymbol{H}$ is the $N \times N$ channel matrix expressed by

$$
\boldsymbol{H}=\left[\begin{array}{ccccccc}
h_{0} & 0 & \cdots & 0 & \cdots & h_{2} & h_{1} \\
h_{1} & h_{0} & \cdots & 0 & \cdots & h_{3} & h_{2} \\
\vdots & \vdots & \ddots & \vdots & \ddots & \vdots & \vdots \\
h_{L-1} & h_{L-2} & \cdots & h_{0} & \cdots & 0 & 0 \\
\vdots & \vdots & \ddots & \vdots & \ddots & \vdots & \vdots \\
0 & 0 & \cdots & h_{L-2} & \cdots & h_{0} & 0 \\
0 & 0 & \cdots & h_{L-1} & \cdots & h_{1} & h_{0}
\end{array}\right],
$$

and $\boldsymbol{n}$ is the $N$-dimensional noise vector.

Transforming (1) into the frequency domain using the $N \times N$ discrete Fourier transform (DFT) matrix $\boldsymbol{F}$ yields

$$
\begin{aligned}
\boldsymbol{F r} & =\boldsymbol{F H} \boldsymbol{s}+\boldsymbol{F n} \\
& =\boldsymbol{F} \boldsymbol{H} \boldsymbol{F}^{H} \boldsymbol{F} \boldsymbol{s}+\boldsymbol{F n} .
\end{aligned}
$$

When the channel is time-invariant within the block, $\boldsymbol{H}$ becomes a cyclic matrix. Then, $\boldsymbol{H}$ can be diagonalized by $\boldsymbol{F}$ as $\boldsymbol{F} \boldsymbol{H} \boldsymbol{F}^{H}=\boldsymbol{D}=\operatorname{diag}\left(d_{1}, \ldots, d_{N}\right)$, so that (4) can be rewritten as

$$
\begin{aligned}
& \boldsymbol{F} \boldsymbol{r}=\boldsymbol{D} \boldsymbol{F} \boldsymbol{s}+\boldsymbol{F} \boldsymbol{n} \\
& \boldsymbol{r}_{F}=\boldsymbol{D} \boldsymbol{s}_{F}+\boldsymbol{n}_{F},
\end{aligned}
$$

where $\boldsymbol{r}_{F}=\boldsymbol{F r}, \boldsymbol{s}_{F}=\boldsymbol{F} \boldsymbol{s}$, and $\boldsymbol{n}_{F}=\boldsymbol{F} \boldsymbol{n}$ are the received signal, the transmitted signal, and the noise vectors represented in the frequency domain, respectively.

The FDE output is given by multiplying $\boldsymbol{r}_{F}$ by an Hermitian transpose of a weight matrix $\boldsymbol{W}$ as

$$
\boldsymbol{y}_{F}=\boldsymbol{W}^{H} \boldsymbol{r}_{F} \text {. }
$$

The optimum weight in the minimum mean square error (MMSE) sense is obtained by minimizing the ensemble average of squared error $\left(\boldsymbol{s}_{F}-\boldsymbol{W}^{H} \boldsymbol{r}_{F}\right)^{H}\left(\boldsymbol{s}_{F}-\boldsymbol{W}^{H} \boldsymbol{r}_{F}\right)$, i.e.,

$$
\boldsymbol{W}=\left(\boldsymbol{D} \boldsymbol{D}^{H}+\sigma^{2} \boldsymbol{I}\right)^{-1} \boldsymbol{D},
$$

where $\sigma^{2}$ denotes the noise power in the frequency domain. (The signal power in the frequency domain is assumed to be 1.) Clearly, the weight matrix calculation is very simple, since $\left(\boldsymbol{D} \boldsymbol{D}^{H}+\sigma^{2} \boldsymbol{I}\right)$ is a diagonal matrix. Finally, applying IFFT into the MMSE-FDE output, we get an estimate of the transmitted signal as $\boldsymbol{y}=\boldsymbol{F}^{H} \boldsymbol{y}_{F}$

\subsection{FDTE}

Turbo equalization is an extremely powerful technique to

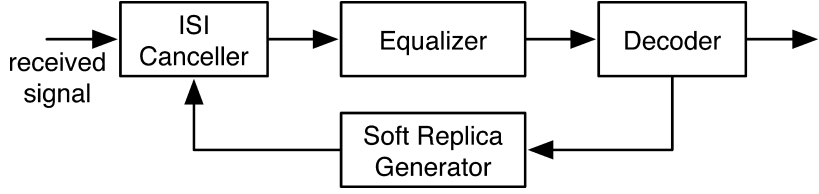

Fig. 1 A concept of turbo equalization.

improve the equalization performance [7]-[10]. This technique is based on an idea exchanging extrinsic information directly or indirectly between an equalizer and a channel decoder as shown in Fig. 1. In the equalizer, soft replica symbols are generated using the extrinsic information provided from the channel decoder and are used to cancel ISI components for target symbol detection.

Turbo equalization can be applied to FDE with some modifications [11]. In the FDTE, the most important thing is to keep a merit of the simplicity in the equalization process. Thus, using some assumptions and approximations, we obtain an estimate of the transmitted signal vector by FDTE as

$$
\boldsymbol{y}_{\mathrm{te}}=(1+\gamma \delta)^{-1}\left\{\gamma \hat{\boldsymbol{s}}+\boldsymbol{F}^{H} \boldsymbol{W}_{\mathrm{te}}^{H} \boldsymbol{F}(\boldsymbol{r}-\boldsymbol{H} \hat{\boldsymbol{s}})\right\},
$$

where $\hat{\boldsymbol{s}}$ is the soft replica vector for $\boldsymbol{s}$ which is calculated using the log-likelihood ratio (LLR) from the received signal [12] and

$$
\begin{aligned}
& \boldsymbol{W}_{\text {te }}=\left\{(1-\delta) \boldsymbol{D} \boldsymbol{D}^{H}+\sigma^{2} \boldsymbol{I}\right\}^{-1} \boldsymbol{D} \\
& \delta=\hat{\boldsymbol{s}}^{H} \hat{\boldsymbol{s}} / N \\
& \gamma=\operatorname{tr}\left(\boldsymbol{W}_{\text {te }}^{H} \boldsymbol{D}\right) / N .
\end{aligned}
$$

In (9), $(\boldsymbol{r}-\boldsymbol{H} \hat{\boldsymbol{s}})$ part means that all soft replicas are cancelled from the received signal. However, the addition of $\gamma \hat{\boldsymbol{s}}$ plays a compensation role for the entire cancellation. It should be noted that the weight matrix in the FDTE still keeps a diagonal matrix form as in (10).

\subsection{Residual Interference Component Due to Fast Fading}

In fast fading environments, cyclicity of the channel matrix $\boldsymbol{H}$ is lost due to channel transition within the block. Thus, $\boldsymbol{F} \boldsymbol{H} \boldsymbol{F}^{H}$ includes non-diagonal components as

$$
\boldsymbol{F} \boldsymbol{H} \boldsymbol{F}^{H}=\boldsymbol{D}^{\prime}+\boldsymbol{E},
$$

where $\boldsymbol{D}^{\prime}$ is the $N \times N$ diagonal matrix, and $\boldsymbol{E}$ is an $N \times N$ matrix of which diagonal elements are zero. Substituting (13) into (4) yields

$$
\boldsymbol{r}_{F}=\boldsymbol{D}^{\prime} \boldsymbol{s}_{F}+\boldsymbol{n}_{F}+\boldsymbol{E} \boldsymbol{s}_{F} .
$$

The third term of this equation corresponds to interfrequency interference (IFI) components (or ISI components in the time domain) due to channel change. Hence, the MMSE estimation is achieved by solving an inverse problem of the $N \times N$ matrix $\left(\boldsymbol{D}^{\prime}+\boldsymbol{E}\right)$, and the numerical complexity grows enormously.

However, if the third term in (14) is relatively small 
compared to the first term, we can still apply FDE or FDTE by regarding the third term as additional noise. Then, (14) is rewritten as

$$
\boldsymbol{r}_{F}=\boldsymbol{D}^{\prime} \boldsymbol{s}_{F}+\boldsymbol{n}_{e, F}
$$

where $\boldsymbol{n}_{e, F}=\boldsymbol{n}_{F}+\boldsymbol{E} \boldsymbol{s}_{F}$. The MMSE weight is solved using $\boldsymbol{D}^{\prime}$ and the equivalent noise $\boldsymbol{n}_{e, F}$. Specifically, the noise covariance matrix $\sigma^{2} \boldsymbol{I}$ in (8) and (10) is replaced by $\boldsymbol{P}_{e}=\operatorname{diag}\left(\sigma_{e, 0}^{2}, \ldots, \sigma_{e, N-1}^{2}\right)$, where $\sigma_{e, k}^{2}$ is an equivalent noise power at the $k$ th frequency point. The approximate calculation for the equivalent noise power is derived in [6].

This equivalent noise assumption is valid when the fading within the block is slow enough. Although the FDTE has a capability to reduce the ISI by soft cancellation, the perfect cancellation can not be expected due to the channel change, i.e., the error in the replica generation, in the fast fading environment. To apply FDE or FDTE under much faster fading environments, therefore, we have proposed the subblock processing described in the next section [5], [6].

\section{FDTE Based on Subblock Processing}

\subsection{Subblock Processing Concept}

In this paper, FDTE utilizes subblock processing. Subblock processing equivalently shortens the FDE block size at the receiver without changing the transmitted block format. The concept is shown in Fig. 2, where ISBI denotes inter-subblock interference from the previous subblock. The basic idea of the subblock processing is dividing the whole received block into $M$ subblocks at the receiver side. As shown in the middle part of Fig. 2, the top $(L-1)$ symbols of each subblock contain ISBI components. Therefore, reduction of the ISBI is required first. In addition, the received signal in the subblock needs to have periodicity so that the tail of the corresponding subblock data convoluted with the multipath channel should be added to the top of the subblock. To satisfy these requirements, the following pseudo CP generation [13] is utilized.

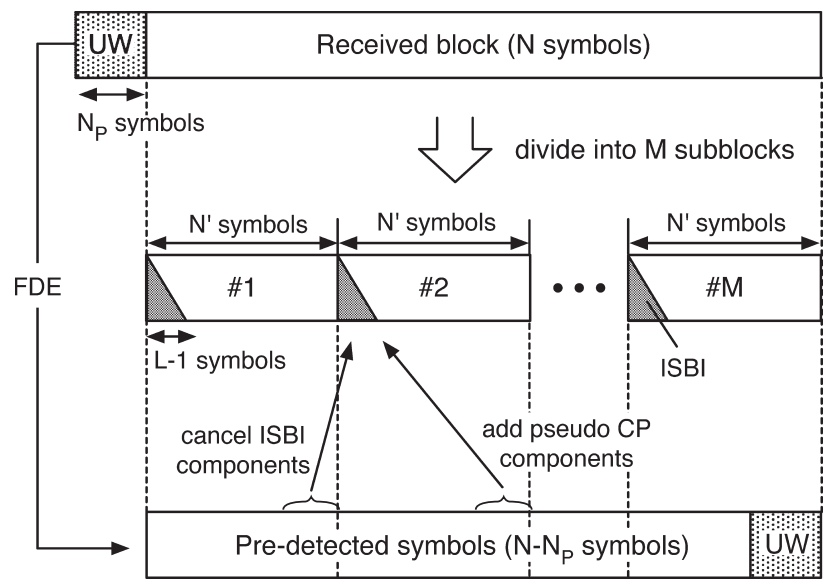

Fig. 2 A concept of basic subblock processing at the receiver side.
At the initial processing, there are no soft replicas yet. Thus, the FDE is first applied to the whole received block for obtaining a soft replica vector $\hat{\boldsymbol{s}}=\left[\hat{s}_{0}, \cdots, \hat{s}_{N-1}\right]^{T}$. We define an $N^{\prime} \times N^{\prime}$ matrix $\boldsymbol{C}_{N^{\prime}}$ as

$$
\boldsymbol{C}_{N^{\prime}}=\left[\begin{array}{cc}
\boldsymbol{O}_{L-1, N^{\prime}-L+1} & \boldsymbol{I}_{L-1} \\
\boldsymbol{O}_{N^{\prime}-L+1, N^{\prime}}
\end{array}\right],
$$

where $N^{\prime}$ is the number of symbols within a subblock (given by $N / M$ ) and $\boldsymbol{O}_{N_{1}, N_{2}}$ is the $N_{1} \times N_{2}$ zero matrix. Then, the $N \times N$ transform matrices $\boldsymbol{T}_{\text {sub }}$ and $\boldsymbol{T}_{\text {add }}$ for cancelling ISBI components and adding pseudo CP components are expressed by

$$
\begin{aligned}
\boldsymbol{T}_{\text {sub }} & =\left[\begin{array}{cccc}
\boldsymbol{O}_{N^{\prime}, N^{\prime}} & \ldots & \boldsymbol{O}_{N^{\prime}, N^{\prime}} & -\boldsymbol{C}_{N^{\prime}} \\
-\boldsymbol{C}_{N^{\prime}} & \ldots & \boldsymbol{O}_{N^{\prime}, N^{\prime}} & \boldsymbol{O}_{N^{\prime}, N^{\prime}} \\
\vdots & \ddots & \vdots & \vdots \\
\boldsymbol{O}_{N^{\prime}, N^{\prime}} & \ldots & -\boldsymbol{C}_{N^{\prime}} & \boldsymbol{O}_{N^{\prime}, N^{\prime}}
\end{array}\right] \\
\boldsymbol{T}_{\mathrm{add}} & =\left[\begin{array}{cccc}
\boldsymbol{C}_{N^{\prime}} & \boldsymbol{O}_{N^{\prime}, N^{\prime}} & \ldots & \boldsymbol{O}_{N^{\prime}, N^{\prime}} \\
\boldsymbol{O}_{N^{\prime}, N^{\prime}} & \boldsymbol{C}_{N^{\prime}} & \ldots & \boldsymbol{O}_{N^{\prime}, N^{\prime}} \\
\vdots & \vdots & \ddots & \vdots \\
\boldsymbol{O}_{N^{\prime}, N^{\prime}} & \boldsymbol{O}_{N^{\prime}, N^{\prime}} & \ldots & \boldsymbol{C}_{N^{\prime}}
\end{array}\right] .
\end{aligned}
$$

With $\boldsymbol{T}_{\text {sub }}$ and $\boldsymbol{T}_{\text {add }}$, the pseudo CP generation is written as

$$
\begin{aligned}
\boldsymbol{r}^{\prime} & =\boldsymbol{r}+\hat{\boldsymbol{H}} \boldsymbol{T}_{\mathrm{sub}} \hat{\boldsymbol{s}}+\hat{\boldsymbol{H}} \boldsymbol{T}_{\mathrm{add}} \hat{\boldsymbol{S}} \\
& =\boldsymbol{r}+\hat{\boldsymbol{H}}\left(\boldsymbol{T}_{\mathrm{sub}}+\boldsymbol{T}_{\mathrm{add}}\right) \hat{\boldsymbol{s}},
\end{aligned}
$$

where $\boldsymbol{r}^{\prime}$ is the $N$-dimensional modified received signal vector composed of $M$ pseudo subblock sequences, and $\hat{\boldsymbol{H}}$ is the $N \times N$ estimated channel matrix.

The pseudo received signal satisfies periodicity within each subblock when the soft replica and the estimated channel are reasonably correct. (The above pseudo CP procedure can be also expressed symbol by symbol as explained in [5].) Thus, the signal now can be equalized by subblockbased FDTE. Each output of the subblock-based FDTE is demultiplexed and passed to a decoder. And, the decoder outputs are fed back to the soft replica generator for the pseudo CP generation and soft cancellation of ISI in the FDTE. Thus, the iteration loop has been achieved.

It should be noted that we use the equivalent noise power even in subblock equalization since the residual interference components still exist after dividing the whole block into small subblocks.

\subsection{Overlapped Layout in Subblock Division}

In this paper, we optionally apply a technique overlapping the subblock layout. The overlapped FDE has been proposed as a method which compensates for non-periodicity in the FDE block [14]. When the periodicity in the FDE block is not satisfied, the major impact appears on symbols at the edges of the block after FDE [15]. Thus, by picking out central data symbols in the block, the effect of non-periodicity can be alleviated.

Figure 3 shows a concept of overlapped layout. As 


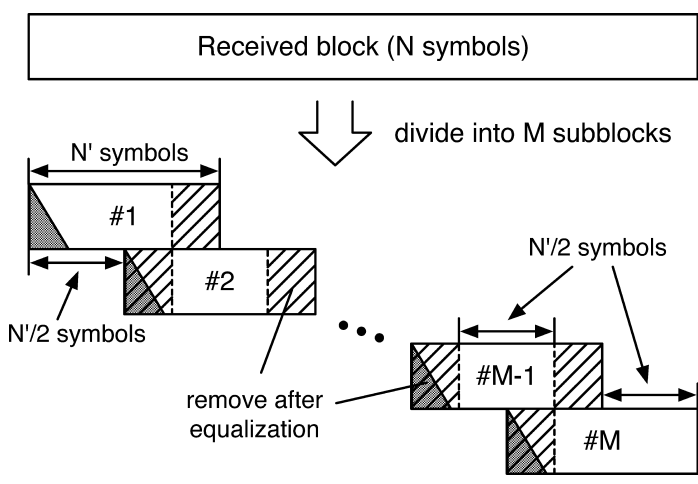

Fig. 3 Overlapped layout.

shown in this figure, each subblock is placed overlapping one another, and then a half of the overlapped symbols in each subblock are just discarded after applying FDTE for the sake of simplicity in our study. In this paper, the overlapped region is set as a half of the subblock length. $N^{\prime}$ is given by $2 N /(M+1)$ in this overlapped layout.

\section{Channel Estimation Using Known Pilot Sequence}

Since we consider a fast fading environment, channel state information (CSI) varying block by block is required at the receiver. The conventional CP (i.e., a copy of the tail in the transmitted block) is basically unknown before detecting data. Therefore, inserting a unique word (UW) as a known pilot sequence instead of the CP has been proposed [16][18]. In this case, the data block size is decreased to $\left(N-N_{P}\right)$ symbols for satisfying periodicity within the FFT window as shown in Fig. 4. When $N_{P} \geq 2 L-1$, a part which is longer than $(L-1)$ symbols and including the known UW only exists in the received signal. Thus, the CSI can be simply estimated exploiting this period.

In our study, the MMSE algorithm for channel estimation in the time domain is applied to this $\left(N_{P}-L\right)$-symbol sequence. In the UW part, using the $\left(N_{P}-L\right)$-dimensional received signal vector $z$, the $L$-dimensional channel vector $\boldsymbol{h}_{\mathrm{uw}}$, and the $\left(N_{P}-L\right) \times L$ transmit signal matrix in this interval $\boldsymbol{Q}$ where the $i$-th column vector is an $i$-symbol-cyclicshifted partial UW sequence, the square error function $J$ is defined as

$$
J=\left(z-\boldsymbol{Q h} \boldsymbol{h}_{\mathrm{uw}}\right)^{H}\left(z-\boldsymbol{Q h} \boldsymbol{h}_{\mathrm{uw}}\right),
$$

where $\boldsymbol{h}_{\mathrm{uw}}$ is assumed as the time-invariant channel response within the target signal sequence. Then, the $\boldsymbol{h}_{\mathrm{uw}}$ can be estimated in the sense of minimizing $J[19]$ as

$$
\boldsymbol{h}_{\mathrm{uw}}=\left(\boldsymbol{Q}^{H} \boldsymbol{Q}\right)^{-1} \boldsymbol{Q}^{H} \boldsymbol{z} .
$$

Finally, the estimated channel response in the time domain is transformed into the frequency domain after zero padding.

We need to know all the channel responses within the block for the processes: i.e., MMSE filtering for all blocks/subblocks, pseudo CP generation, and soft cancellation in the time domain, as described later. Since the UW

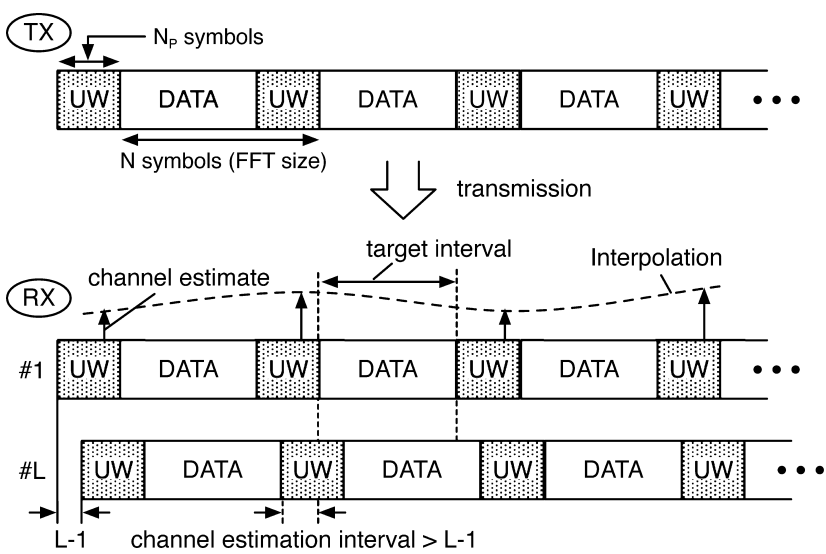

Fig. 4 Transmitted signal block and channel estimation.

for channel estimation is located only at pre- and post-data blocks, we consider to obtain channel estimates in the data block by interpolation. In this paper, third-order interpolation is used as illustrated in Fig. 4. By using channel estimates at four UWs (two in the past and two in the future of the target data block), the channel within the data block is interpolated with a cubic function (see subsection 3-1 in [20] for the detail).

When applying the MMSE filtering in the frequencydomain, we used the central channel estimate $\boldsymbol{h}_{\text {cent }}=$ $\left[h_{0, \text { cent }}, \ldots, h_{L-1, \text { cent }}, 0, \ldots, 0\right]^{T}$ in the target block to calculate $d_{0}, \ldots, d_{N-1}$, i.e., $\left[d_{0}, \ldots, d_{N-1}\right]^{T} \simeq \boldsymbol{F} \boldsymbol{h}_{\text {cent }}$. For the pseudo CP generation, the interpolated channel estimate at each of top $(L-1)$ symbols in the target subblock is utilized.

The soft cancellation in (9) can be done with the timeinvariant and periodic channel matrix as well as the MMSE filtering since the FDTE processing is based on that nature. In addition, the cancellation in the frequency domain achieves lower computational complexity. However, to improve the soft cancellation accuracy, we apply the timedomain cancellation with the time-variant channel matrix provided by the interpolation in the following study.

\section{Numerical Analysis}

\subsection{Simulation Environment}

The performance of the proposed system was numerically evaluated using computer simulations. The simulation parameters are listed in Table 1. In the following discussions, we use the normalized Doppler frequency $F_{D}$, which is a product of the maximum Doppler frequency $f_{D}$ and the block length $N T_{s}$ ( $T_{s}$ : the symbol duration), as a fading speed measure.

The receiver structure is shown in Fig. 5. We set the maximum iteration number in the FDTE to five since the further increase of iterations did not yield a significant improvement. In addition, the packet data transmission with ARQ based on an error detection is assumed. Therefore, as shown in this figure, once no errors in the cyclic redundancy check (CRC) are found, the iteration is terminated. For the 
Table 1 Simulation parameters.

\begin{tabular}{c|c}
\hline \hline modulation & QPSK for data (BPSK for UW) \\
\hline block size $N$ & $\begin{array}{c}256 \text { symbols } \\
\text { (data: 208 symbols) } \\
\text { (UW: 48 symbols) }\end{array}$ \\
\hline number of received antennas & $1,2,4$ \\
\hline number of paths $L$ & 16 (symbol-spaced, equal-level) \\
\hline fading model & $\begin{array}{c}\text { Rayleigh (Jakes' model) } \\
\text { uncorrelated between paths }\end{array}$ \\
\hline number of subblocks $M$ & $\begin{array}{c}2,4,8 \text { (conventional layout) } \\
3,7,15 \text { (overlapped layout) }\end{array}$ \\
\hline FEC & binary CC (4 states, $\left.R_{c} 1 / 2\right)$ \\
\hline decoder & Max log-MAP \\
\hline maximum number of & 5 \\
turbo iterations & 100,000 \\
\hline number of transmitted blocks &
\end{tabular}

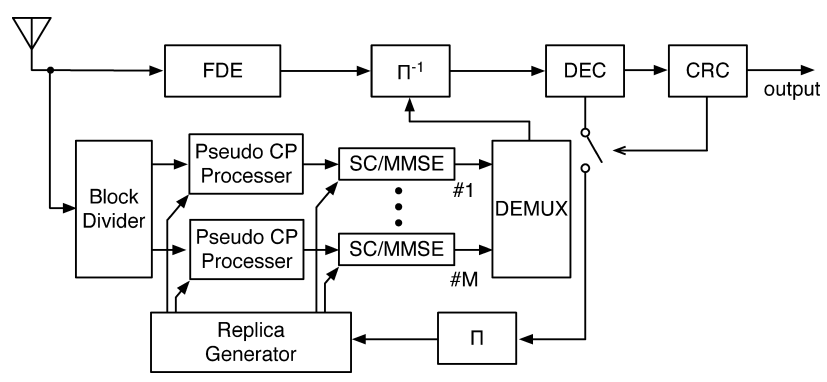

Fig. 5 A structure of subblock based FDTE.

sake of convenience, we assume the perfect error detection without using the actual CRC code. The perfect synchronization for DFT windowing is also assumed.

\subsection{BLER Performance of FDTE Based on Subblock Pro- cessing}

First, let us evaluate the performance of the FDTE with and without subblock processing. Figure 6 shows a block error rate (BLER) performance, where the dashed and solid curves denote the case of perfectly known CSI and estimated CSI, respectively. And, the case of "no division" shows the performance of the FDTE without subblock processing.

In the case of $F_{D}=0.3$, as shown in Fig. 6(a), the sufficient performance can be obtained without subblock processing. However, the performance in $F_{D}=0.4$ shows error floors in a high SNR region in the estimated CSI case. In these results, although the IFI due to fast fading can be reduced by using FDTE, the channel change in the case of $F_{D}=0.4$, where the maximum phase rotation within a block of 256 symbols reaches 144 degrees, is very large so that the IFI cannot be properly reduced.

By using two-subblock processing, such error floors cannot be seen, and the best performance is obtained as shown in Fig. 6(b). The channel variation within a subblock becomes slower with smaller subblock size. Thus,

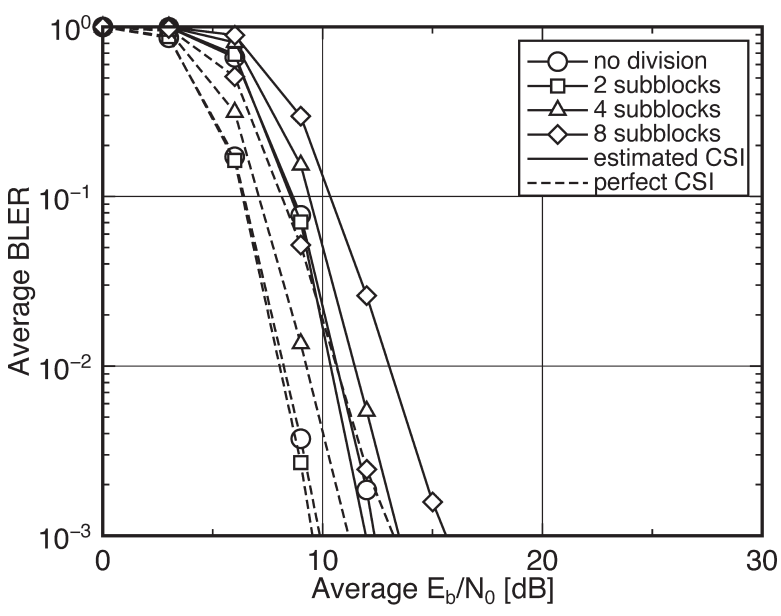

(a) $F_{D}=0.3$

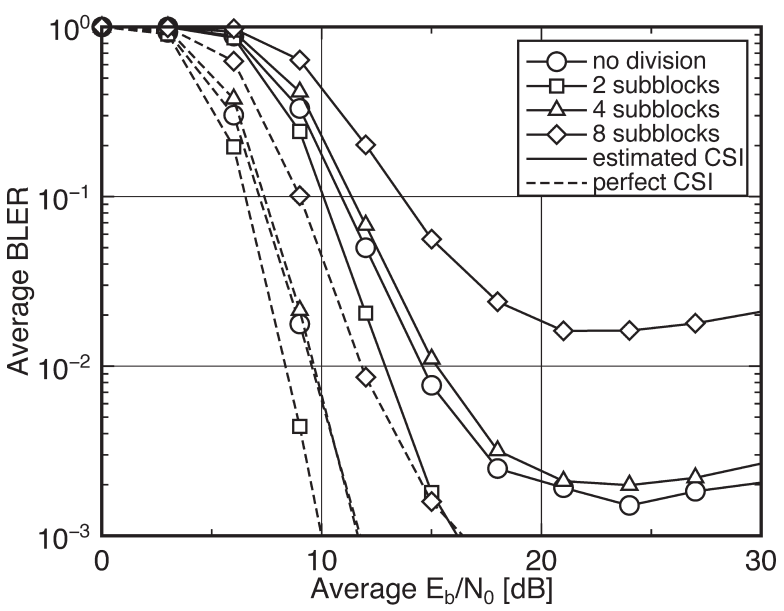

(b) $F_{D}=0.4$

Fig. 6 BLER performance of FDTE based on subblock processing (one received antenna).

the smaller subblock size is expected to be more tolerant to $F_{D}$ in principle. However, the proposed method is affected by the accuracy of the pseudo $\mathrm{CP}$ which is composed of the channel estimate and soft replicas. Since the smaller subblock size makes the ratio of the pseudo CP to the subblock size higher, the BLER performances in the four- and eight-subblock cases are degraded due to the high pseudo CP ratio.

Considering that the two-subblock processing shows the best performance even in the perfectly-known channel case, we can say that the accuracy of soft replicas for pseudo $\mathrm{CP}$ generation is insufficient for smaller subblock cases. The $\mathrm{CP}$ at the first iteration is produced from the normal FDE output. When we assume the random errors in the decoded sequence, the inserted errors due to the pseudo CP increases with the number of subblocks. On the other hand, the turbo equalization is a non-linear process. When the number of errors in the received signal is too many, the turbo process tends to increase the errors. This is the reason that the twosubblock case was the best in Fig. 6 .

However, the above result is not true when the block 


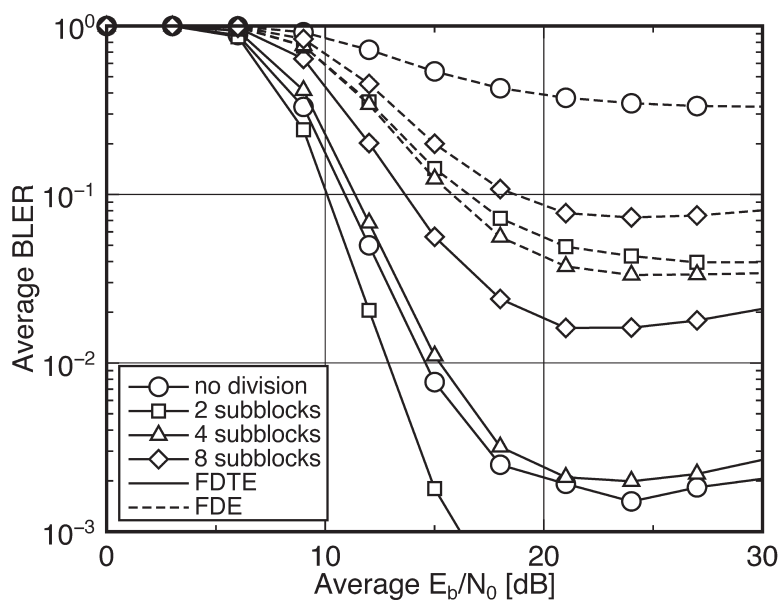

(a) $F_{D}=0.4$

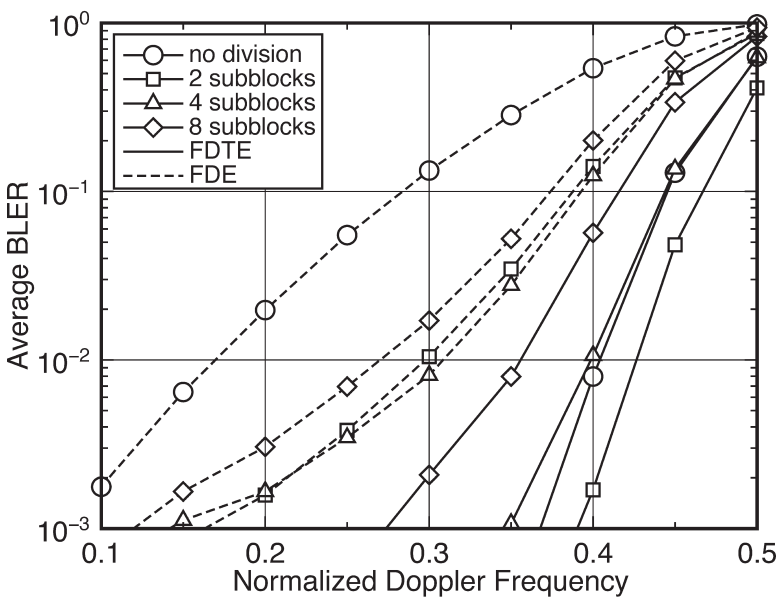

(b) Average $E_{b} / N_{0}=15 \mathrm{~dB}$

Fig. 7 BLER performance in the FDTE and FDE (one received antenna).

size changes. For example, the ratio of inserted errors in the subblock in the case of $N=1024$ and $M=8$ is similar to the one in the case of $N=256$ and $M=2$. Thus, if the block size is larger than this study, the optimum number of subblock may change [6]. Optimizing the number of subblocks is an important issue to be solved. However, it needs lots of data for various fading speeds and block sizes. The optimization should be a most urgent study in the near future.

In the case of $F_{D}=0.4$, the degradation due to the channel estimation error is about $4 \mathrm{~dB}$ at a BLER of $1.0 \times 10^{-2}$ in the two-subblock processing. More accurate channel estimation/interpolation should be needed to reduce the gain loss. It should be noted that, in the estimated CSI case, a rebound of error floors in the four- and eight-subblock and no division cases occurs due to underestimation of the equivalent noise power [6].

\subsection{Comparison to Subblock Based FDE}

Next, we compare the FDTE performance with the conventional FDE when applying subblock processing. Figure 7(a)

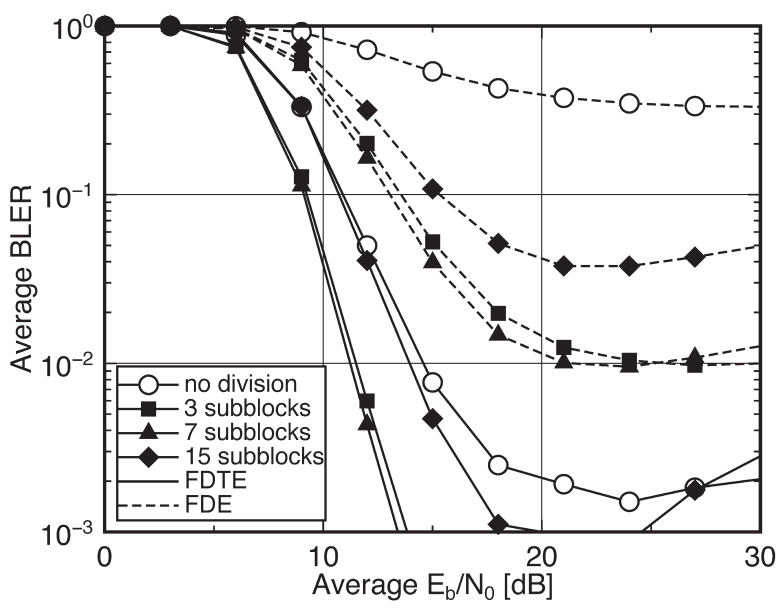

(a) $F_{D}=0.4$

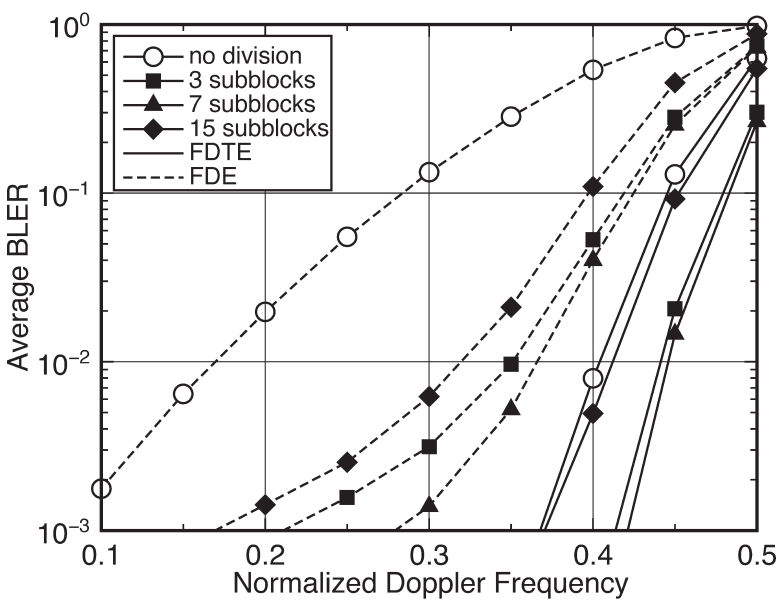

(b) Average $E_{b} / N_{0}=15 \mathrm{~dB}$

Fig. 8 BLER performance for overlapped layout in the FDTE and FDE (one received antenna).

shows the BLER performance for $F_{D}=0.4$ (the same as Fig. 6(b) for the FDTE performance) where the estimated channel only is used. In the figure, dashed and solid curves denote the cases of FDE and FDTE, respectively.

The performance improvement by turbo equalization is remarkable. In the conventional FDE case, the performance without subblock processing shows a high error floor at BLER $=3.0 \times 10^{-1}$. However, by using FDTE, the error floor can be reduced by about $1 / 100$ of that. This means that FDTE can suppress ISI more strongly compared with conventional FDE so that we can obtain more accurate replicas with FDTE. Then, when the subblock processing is applied, no error floor is observed in the two-subblock processing case with FDTE, whereas the least error floor in the FDE is about $3.5 \times 10^{-2}$. It can be said that the turbo equalization effectively improves the accuracy of pseudo CP generation and ISI cancellation, especially in the two-subblock case.

Figure 7(b) shows the BLER performance in a range of $0.1 \leq F_{D} \leq 0.5$ for a fixed average $E_{b} / N_{0}$ of $15 \mathrm{~dB}$. The result also clearly indicates the effectivity of turbo equalization. When assuming a required BLER of $10^{-2}$, with FDTE 


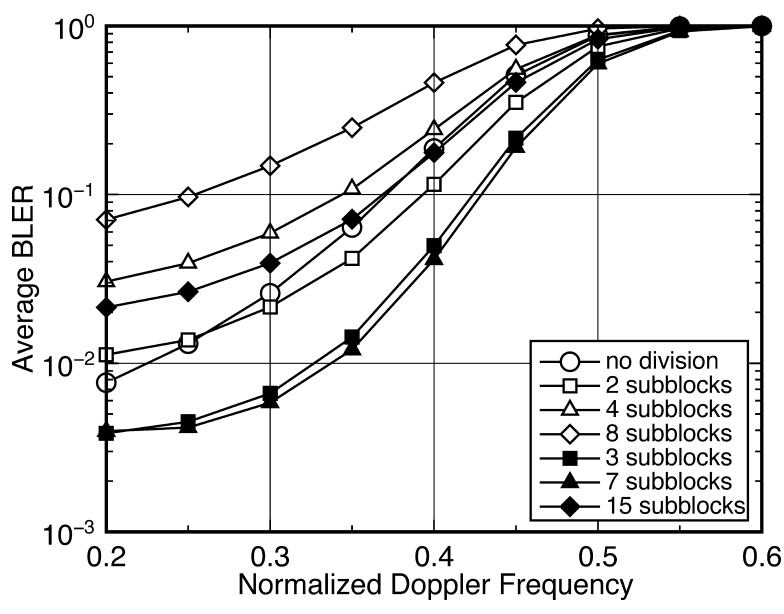

(a) One received antenna

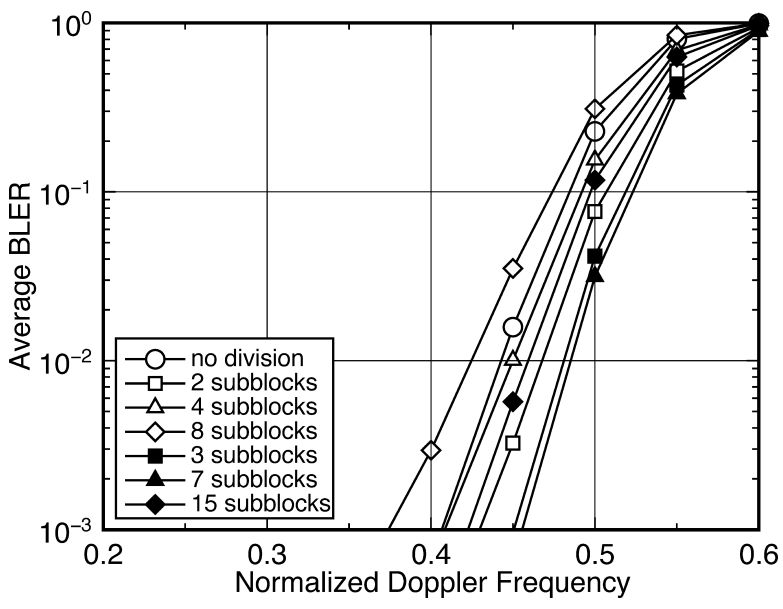

(b) Two received antennas

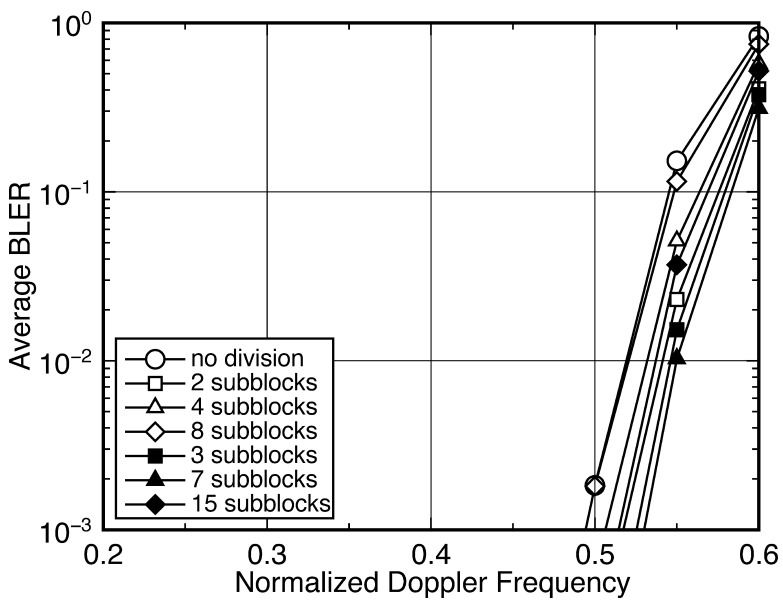

(c) Four received antennas

Fig. 9 BLER performance using multiple received antennas when average $E_{b} / N_{0}=10 \mathrm{~dB}$.

two-subblock processing is applicable until $F_{D}=0.42$, which corresponds to about 1.4 times the speed applicable in the conventional FDE with four-subblock processing, i.e., $F_{D}=0.31$.

Next, we evaluate the effect of overlapped subblock layout using its BLER performance shown in Fig. 8. We see that the overlapped layout clearly improves the performance of FDTE. To be specific, Fig. 8(b) shows that the threeand seven-subblock layouts are applicable up to $F_{D}=0.44$ and $F_{D}=0.45$, respectively, when the required BLER $=1.0 \times 10^{-2}$. Considering that the increase of complexity in the three-subblock processing is only about 1.5 -fold compared to the two-subblock case, we can say that the overlapped layout is an attractive approach to easily improve the FDTE performance.

\subsection{BLER Performance Using Multiple Received Anten- nas}

Finally, we evaluate the performance in the case of multiple received antennas for more performance improvement. See [11] for details on the FDTE algorithm in the case of multiple-antenna reception. Figure 9 shows the BLER performance versus $F_{D}$ when $E_{b} / N_{0}=10 \mathrm{~dB}$. Compared with the case of one received antenna, it can be seen that the performance is significantly improved when two received antennas are used. Moreover, in the case of four received antennas, the three-subblock processing is applicable until $F_{D}=0.55$, which corresponds to about 1.6 times the speed of the one-received-antenna case, i.e., $F_{D}=0.35$. Consequently, with diversity reception, we can compensate for the performance degradation due to errors in soft replicas and channel estimates.

It should be noted that the best performance is obtained by the FDTE without subblock processing for less than $F_{D}=0.25$ in Fig. 9(a). The degradation in the twosubblock processing case is caused by errors in the pseudo $\mathrm{CP}$ in a low SNR condition. In such a case, the degradation due to the channel change in the FDTE without subblock processing seems to be relatively small.

\section{Conclusion}

In this paper, we have proposed the application of subblock processing to FDTE. The numerical analysis has shown that the performance can be improved remarkably, so that applying FDTE to subblock processing is very effective. It has also been shown that the $F_{D}$ can reach 0.55 and still maintain a BLER of $1.0 \times 10^{-2}$ using four received antennas and overlapped layout. However, at very high Doppler frequency, the accuracy of the channel estimation degrades. Therefore, reduction of the channel estimation error should be part of further studies. In addition, the optimum number of subblocks in the various block size should also be discussed in the future.

\section{References}

[1] L.J. Cimini, Jr., "Analysis and simulation of a digital mobile channel using orthogonal frequency division multiplexing," IEEE Trans. Commun., vol.33, no.7, pp.665-675, July 1985.

[2] K.G. Paterson and V. Tarokh, "On the existence and construction of good codes with low peak-to-average power ratios," IEEE Trans. Inf. Theory, vol.46, no.6, pp.1974-1987, Sept. 2000. 
[3] D. Falconer, S.L. Ariyavisitakul, A. Benyamin-Seeyar, and B. Eidson, "Frequency domain equalization for single-carrier broadband wireless systems," IEEE Commun. Mag., vol.40, no.4, pp.58-66, April 2002.

[4] A. Okada, S. Ibi, and S. Sampei, "A study on frequency-domain SC/MMSE with adaptive frame control technique," IEICE Technical Report, RCS2006-4, April 2006.

[5] K. Kambara, H. Nishimoto, T. Nishimura, Y. Ogawa, and T. Ohgane, "Subblock processing for MMSE-FDE under fast fading environments," Proc. VTC 2007-Spring, pp.2190-2194, April 2007.

[6] K. Kambara, H. Nishimoto, T. Nishimura, T. Ohgane, and Y. Ogawa, "Subblock processing in MMSE-FDE under fast fading environments," IEEE J. Sel. Areas Commun., vol.26, no.2, pp.359-365, Feb. 2008.

[7] D. Reynolds and X. Wang, "Low-complexity turbo-equalization for diversity channels," Signal Process., vol.81, no.5, pp.989-995, May 2001.

[8] M. Tuchler, R. Koetter, and A.C. Singer, "Turbo equalization: Principles and new results," IEEE Trans. Commun., vol.50, no.5, pp.754-767, May 2002.

[9] T. Abe and T. Matsumoto, "Space-time turbo equalization in frequency-selective MIMO channels," IEEE Trans. Veh. Technol., vol.52, no.3, pp.469-475, May 2003.

[10] S. Ibi, T. Matsumoto, S. Sampei, and N. Morinaga, "EXIT chartaided adaptive coding for MMSE turbo equalization with multilevel BICM,” IEEE Commun. Lett., vol.10, no.6, pp.486-488, June 2006.

[11] K. Kansanen, Wireless Broadband Single-Carrier Systems with MMSE Turbo Equalization Receivers, Ph. D. Thesis, University of Oulu, Dec. 2005

[12] N. Miki, S. Abeta, H. Atarashi, and M. Sawahashi, "Multipath interference canceller using soft-decision replica combined with hybrid ARQ in W-CDMA forward link," Proc. VTC2001-Fall, pp.19221926, Oct. 2001.

[13] T. Hwang and Y. (G.) Li, "Iterative cyclic prefix reconstruction for coded single-carrier systems with frequency-domain equalization (SC-FDE)," Proc. VTC2003-Spring, vol.3, pp.1841-1845, April 2003

[14] I. Martoyo, T. Weiss, F. Capar, and F.K. Jondral, "Low complexity CDMA downlink receiver based on frequency domain equalization," Proc. IEEE VTC2003-Fall, pp.987-991, Sept./Oct. 2003.

[15] H. Tomeba, K. Takeda, and F. Adachi, "Overlap MMSE-frequencydomain equalization for multi-carrier signal transmission," Proc. WPMC2006, pp.751-755, Sept. 2006.

[16] L. Deneire, B. Gyselinckx, and M. Engels, "Training sequence versus cyclic prefix - A new look on single carrier communication,' IEEE Commun. Lett., vol.5, no.7, pp.292-294, July 2001.

[17] H. Witschnig, T. Mayer, A. Springer, A. Koppler, L. Maurer, M. Huemer, and R. Weigel, "A different look on cyclic prefix for SC/FDE,” Proc. PIMRC'02, vol.2, pp.824-828, Sept. 2002.

[18] J. Coon, M. Sandell, M. Beach, and J. McGeehan, "Channel and noise variance estimation and tracking algorithms for unique-word based single-carrier systems," IEEE Trans. Wirel. Commun., vol.5, no.6, pp.1488-1496, June 2006.

[19] Y. Ogawa, K. Nishio, T. Nishimura, and T. Ohgane, "A MIMOOFDM system for high-speed transmission," Proc. VTC2003-Fall, vol.2, pp.1523-1527, Sept. 2003.

[20] W.H. Press, B.P. Flannery, S.A. Teukolsky, and W.T. Vetterling, Numerical Recipes in C: The Art of Scientific Computing, second ed., Cambridge University Press, 1992.

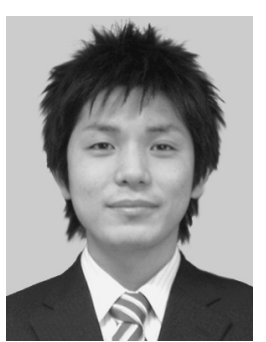

Keiichi Kambara received the B.E. degree from Hokkaido University, Sapporo, Japan, in 2006. He is currently a graduate student toward the M.E. degree at the Graduate School of Information Science and Technology, Hokkaido University. His primary interests include high-mobility wireless systtems and multiuser-MIMO systems. Mr. Kambara received the IEEE VTS Japan Chapter Young Researcher's Encouragement Award in 2007.

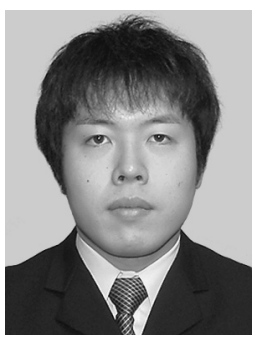

Hiroshi Nishimoto received the B.E. M.E., Ph.D. degrees from Hokkaido University, Sapporo, Japan, in 2003, 2005, and 2007, respectively. $\mathrm{He}$ is currently a Post-Doctoral Fellow at the Graduate School of Information Science and Technology, Hokkaido University. His research interests are in MIMO propagation measurement and MIMO communication systems. He has been a Research Fellow of the Japan Society for the Promotion of Science since 2005. He received the IEEE VTS Japan Chapter Student Paper Award and the Young Researchers' Award of IEICE Japan, both in 2007, and the 2006 IEICE RCS Active Research Award. Dr. Nishimoto is a member of the IEEE.

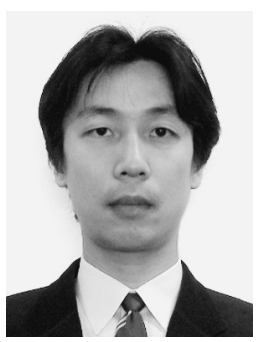

Toshihiko Nishimura received the B.S and M.S. degrees in physics and Ph.D. degree in electronics engineering from Hokkaido University, Sapporo, Japan, in 1992, 1994, and 1997 respectively. In 1998, he joined the Graduate School of Engineering (reorganized to Graduate School of Information Science and Technology at present) at Hokkaido University, where he is currently an Assistant Professor of the Graduate School of Information Science and Technology. His current research interests are in MIMO systems using smart antenna techniques. Dr. Nishimura received the Young Researchers' Award of IEICE Japan in 2000, and the Best Paper Award from IEICE Japan in 2007. Dr. Nishimura is a member of the IEEE.

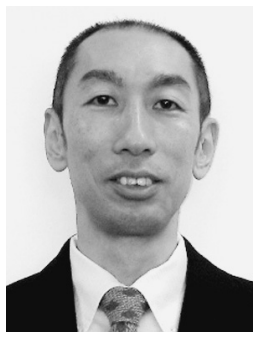

Takeo Ohgane received the B.E., M.E., and $\mathrm{Ph} . \mathrm{D}$. degrees in electronics engineering from Hokkaido University, Sapporo, Japan, in 1984, 1986, and 1994, respectively. From 1986 to 1992, he was with Communications Research Laboratory, Ministry of Posts and Telecommunications. From 1992 to 1995, he was on assignment at ATR Optical and Radio Communications Research Laboratory. Since 1995, he has been with Hokkaido University, where he is an Associate Professor. During 2005-2006, he was at Centre for Communications Research, University of Bristol, U.K., as a Visiting Fellow. His interests are in MIMO signal processing for wireless communications. Dr. Ohgane received the IEEE AP-S Tokyo Chapter Young Engineer Award in 1993, the Young Researchers' Award of IEICE Japan in 1990, and the Best Paper Award from IEICE Japan in 2007. Dr. Ohgane is a member of the IEEE. 


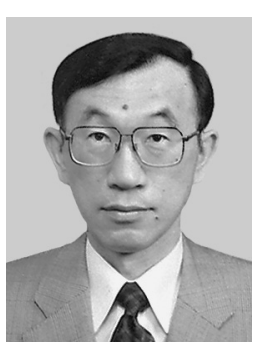

Yasutaka Ogawa received the B.E., M.E. and Ph.D. degrees from Hokkaido University, Sapporo, Japan, in 1973, 1975, and 1978, respectively. Since 1979, he has been with Hokkaido University, where he is currently a Professor of the Graduate School of Information Science and Technology. During 1992-1993, he was with ElectroScience Laboratory, the Ohio State University, U.S.A., as a Visiting Scholar, on leave from Hokkaido University. His interests are in adaptive antennas, mobile communications, super-resolution techniques, and MIMO systems. Dr. Ogawa received the Yasujiro Niwa outstanding paper award in 1978, the Young Researchers' Award of IEICE Japan in 1982, and the Best Paper Award from IEICE Japan in 2007. Dr. Ogawa is a senior member of the IEEE. 